\title{
CDISC Functional Test Terminology
}

National Cancer Institute

\section{Source}

National Cancer Institute. CDISC Functional Test Terminology. NCI Thesaurus. Code C115302.

The terminology subset that includes terms pertaining to the CDISC functional test terminology. 